\title{
Numerical simulation of time-invariant error and its effect on planetary gearbox dynamics
}

\author{
Nithin Venkataram ${ }^{1 *}$, Rahul M. Cadambi ${ }^{1}$, and Arun R. Rao ${ }^{1}$ \\ ${ }^{1}$ Dept. of Mechanical and Manufacturing Engineering, Ramaiah University of Applied Sciences, \\ Bangalore, India
}

\begin{abstract}
Planetary gearbox is used in high precision applications such as robotic arm, control system of antenna, positioning and radar tracking systems. Planetary gearbox have high torque-to-weight ratio, high degree of control over the speed range and better efficiency. Most of the literatures assume that the gearbox are free from errors. Errors significantly affect the dynamic characteristics of the gearbox. The major challenge is to model these errors and study its behaviour under dynamic condition. The simulation results of time domain signal when converted to frequency domain signal, it shows the presence of error in the gearbox. Also, simulation result indicates a non-uniform motion of planetary gearbox in the presence of errors.
\end{abstract}

\section{Introduction}

In applications such as position control system in robotics, radars, antenna and tracking system, the most important criteria is speed reduction and torque amplification. Generally in these applications, there are numerous problem related to accuracy in controlling and positioning. Although servo motors are in use, their applications are limited due to no torque amplification. These drawbacks can be overcome by the use of mechanical gear drives. These mechanical gear drives has a major advantage of speed reduction and torque amplification.

In recent years, planetary gearbox has received great attention when compared to fixed axis gearbox. In fixed axis gearbox, gears revolve about an axis and only one speed reduction ratio is achieved. In addition to this, in fixed axis gearbox, the power to weight ratio is comparatively low. To overcome these shortcomings, the planetary gearbox is used. In addition to this, just by increasing the number of planet gears in planetary gearbox, the load carrying capacity drastically increases. Also, the torsional rigidity is higher than other fixed axis gearbox. In addition, it is possible to obtain variable speed reduction by fixing either sun, planet or ring gear.

The challenge lies in addressing issues related to improving the accuracy of the position control system and its dynamics. In dynamic condition, when gears in the planetary gear meshes, it transfers uniform angular motion and torque, which is possible only in an ideal

\footnotetext{
*Corresponding author: nithin31@gmail.com
} 
case. The main reason for the deviation from ideal condition is due to errors present in the system.

In dynamic condition, contact loss is one of the important parameter to be analysed [1] for tuned and mistuned planetary gearbox. The contact loss between the two spur gear pair is studied through non-linear dynamic analysis [2] through experimental work. A model is developed to study the influence of damping co-efficient and vibrational parameters of a gearbox system [3]. The gearbox, which is coupled to speed control drive unit introduces speed change and so does the change in frequency and vibration characteristics. These vibration characteristics are analysed by condition monitoring of planetary gearbox to identify errors under dynamic conditions [4]. In addition to these, the dynamic response of the planetary gear significantly changes with stiffness of the pins [5] and eccentricity of gears [6].

Most of the literature assumes that the gearboxes are free from any defect or errors. But in actual condition the planetary gearbox are not free from defects. The challenge lies in identifying these errors in planetary gearbox under dynamic conditions. This paper focuses on modelling of errors associated with gear manufacturing and its effect on planetary gearbox dynamics.

\section{Planetary gearbox design}

The majority of the high precision application in which planetary gearbox are used have a speed reduction and torque amplification. To analyse the effect of errors in a planetary gearbox, a single stage planetary gearbox with 5:1 reduction ratio is designed from the first principle. The line diagram of the designed planetary gearbox is shown in Fig. 1.

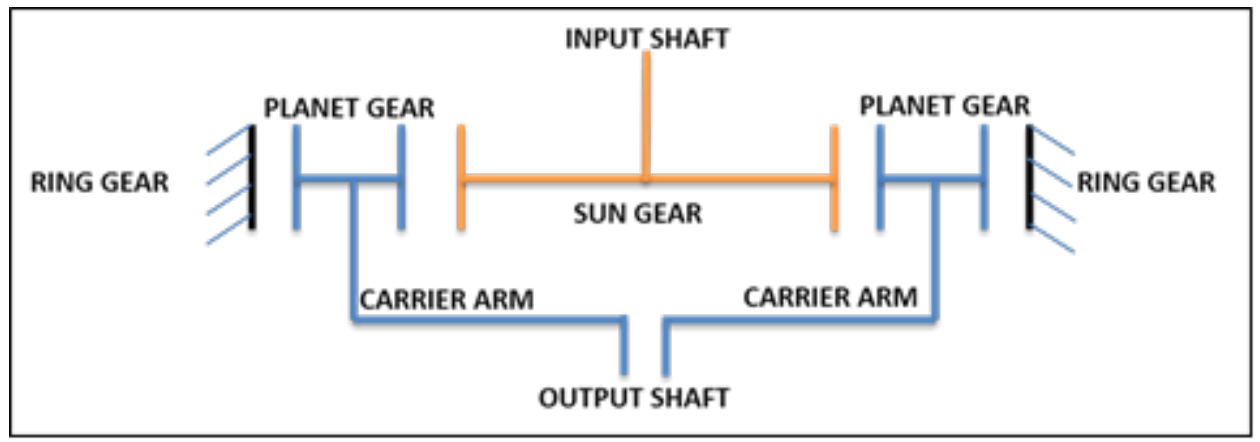

Fig. 1. Line diagram of a single stage planetary gearbox

The designed planetary gearbox consists of a sun gear which is coupled to input shaft. Around sun gear, three planet gears revolve and simultaneously meshes with internal ring gear. These three planet gears are connected to carrier arm which is coupled to output shaft of the planetary gearbox. The planetary gearbox parameters are tabulated in table 1 .

Table 1. Planetary gearbox parameter

\begin{tabular}{|l|l|l|l|}
\hline Parameter & Sun gear & Planet gear & Ring Gear \\
\hline No. of teeth & 24 & 36 & 96 \\
\hline Module & 1 & 1 & 1 \\
\hline Pressure angle (degree) & 20 & 20 & 20 \\
\hline Pitch circle diameter $(\mathrm{mm})$ & 24 & 36 & 98.5 \\
\hline Root diameter $(\mathrm{mm})$ & 21.5 & 36.75 & 94 \\
\hline
\end{tabular}




\section{DIN gear standards}

Errors are classified into three categories: time-invariant assembly error, time-invariant assembly dependent error and time-variant assembly dependent error [7].

The planetary gearbox and its components are manufactured as per DIN standards. This section provides details about the various manufacturing tolerance level that can be achieved. As per DIN 3961, the tolerance level is considered from functionality and manufacturing point of view. The functionality is sub-classified based on uniformity of transmission of motion, quiet running and static load carrying capacity. For application such as position control system, antenna and radar tracking system the main functionality is uniformity of transmission of motion. As per DIN 3961 [8], the critical errors that are responsible for non-uniformity in transmission of motion are single flank, two flank and total pitch errors. Among these errors, the effect of single flank error is identified as it is a time-invariant error. Therefore, the present paper focus is on analysing single flank error on planetary gearbox under dynamic condition. Considering the gear parameters from table 1 , single flank error is calculated as,

$$
\begin{aligned}
& \text { Profile form deviation }=1.5+0.25 *\left(\mathrm{~m}_{\mathrm{n}}+9 * \sqrt{ } \mathrm{m}_{\mathrm{n}}\right) \\
& \text { Profile angle deviation }=2.5+0.25 *\left(\mathrm{~m}_{\mathrm{n}}+3 * \sqrt{ } \mathrm{m}_{\mathrm{n}}\right) \\
& \text { Total pitch deviation }=7.25 *\left(\mathrm{~d}^{(1 / 3)} / \mathrm{z}^{(1 / 7)}\right)
\end{aligned}
$$

Total profile deviation $=\sqrt{ }(\text { profile angle deviation })^{2}+(\text { profile form deviation })^{2}$

$$
\text { Single flank error }=0.8 *(\text { Toltal pitch deviation }+ \text { Total profile deviation })
$$

Substituting the value of reference diameter (d) for planet gear as 36, number of teeth (z) as 36 and module (m) as $1 \mathrm{~mm}$ in eqn. 1 to 5 [8], the single flank error is found to be 43 $\mu \mathrm{m}$ for class 8 gear quality.

\section{Modelling and rigid body simulation of single flank error}

In previous section, value of single flank error is calculated through numerical calculation. In this section, single flank error is modelled on a single planet gear and its effect under dynamic condition is simulated. The modelled single flank error on a planet is shown in Fig. 2. The error is indicated by measuring at pitch circle diameter i.e. $(1.437-0.043=1.395$ $\mathrm{mm})$.

Planetary gearbox components are modelled and assembled in Solidworks 2012. Model is imported into MSc ADAMS 2013 environment for the simulation and is shown in Fig. 3. Contact properties between the sun, planet and ring gear are modelled in ADAMS [9]. In ADAMS the ring gear is fixed, revolutary joint is provided for sun, ring and carrier of the planetary gear. The input motion of $104.7 \mathrm{rad} / \mathrm{s}$ is provided for the sun. Simulation is carried out for 1 second and 2000 steps.

From the simulation, the time domain signal of contact force vs. time is plotted and is shown in Fig. 4. The plot shows the variation of contact force between the sun and planet gears. The variation of force is due to engaging and disengaging of gear tooth. 


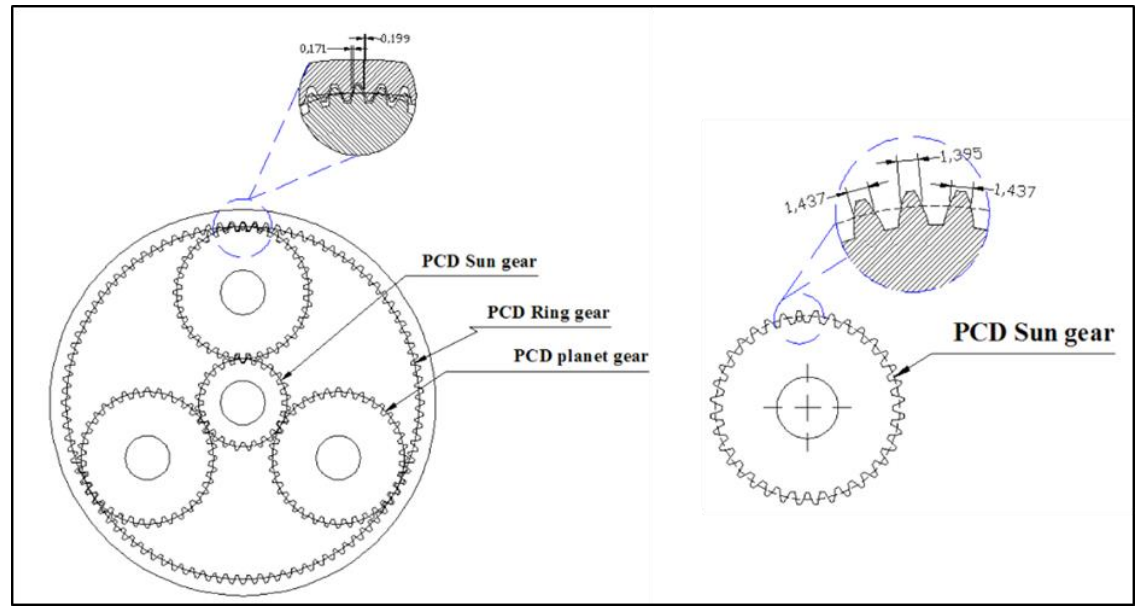

Fig. 2. Modelling of single flank error on a planet of a planetary gearbox

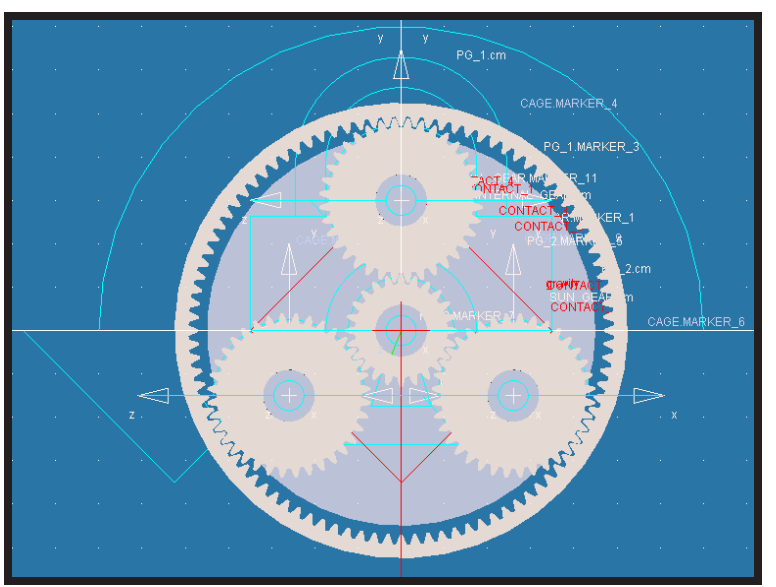

Fig. 3. Constraints for the error induced planetary gearbox in ADAMS

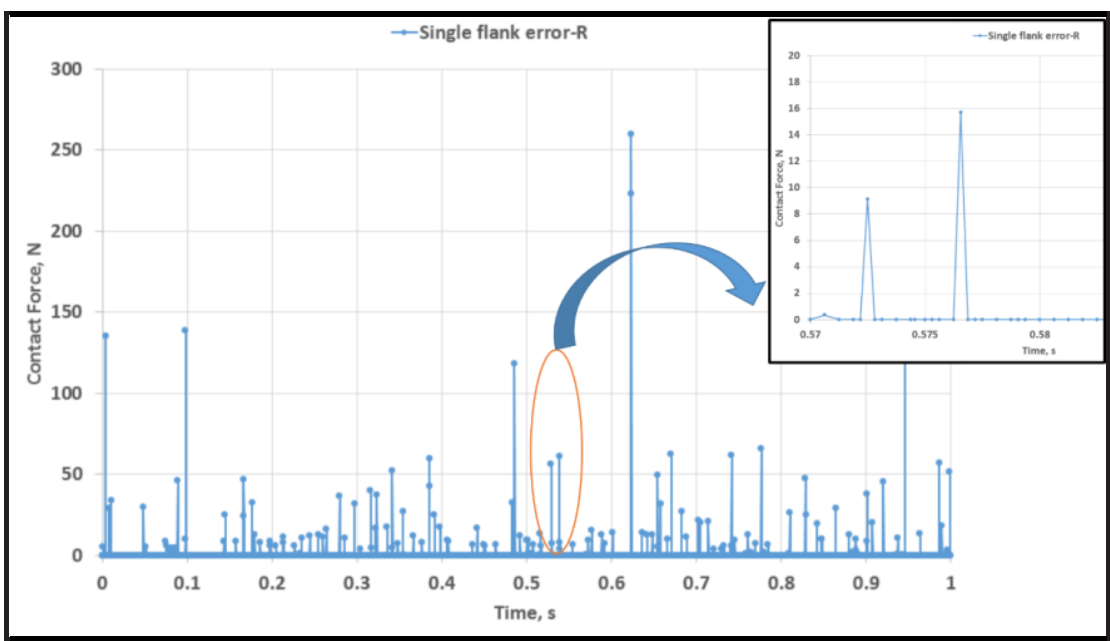

Fig. 4. Contact force variation of single flank error induced planetary gearbox 
This time domain signal of contact force vs. time is converted to frequency domain signal for mesh frequencies and its harmonics through an FFT analysis. Fig. 5, shows the mesh frequency and its harmonics of the error induced planetary gearbox. The presence of these errors does not cause any change in mesh frequencies. Frequencies are plotted up to $1000 \mathrm{~Hz}$ and gear meshing frequencies are indicated by three distinct peak at $320 \mathrm{~Hz}, 640$ $\mathrm{Hz} \& 960 \mathrm{~Hz}$. The presence of error is indicated by sidebands around meshing frequencies which are presented in Fig. 6.

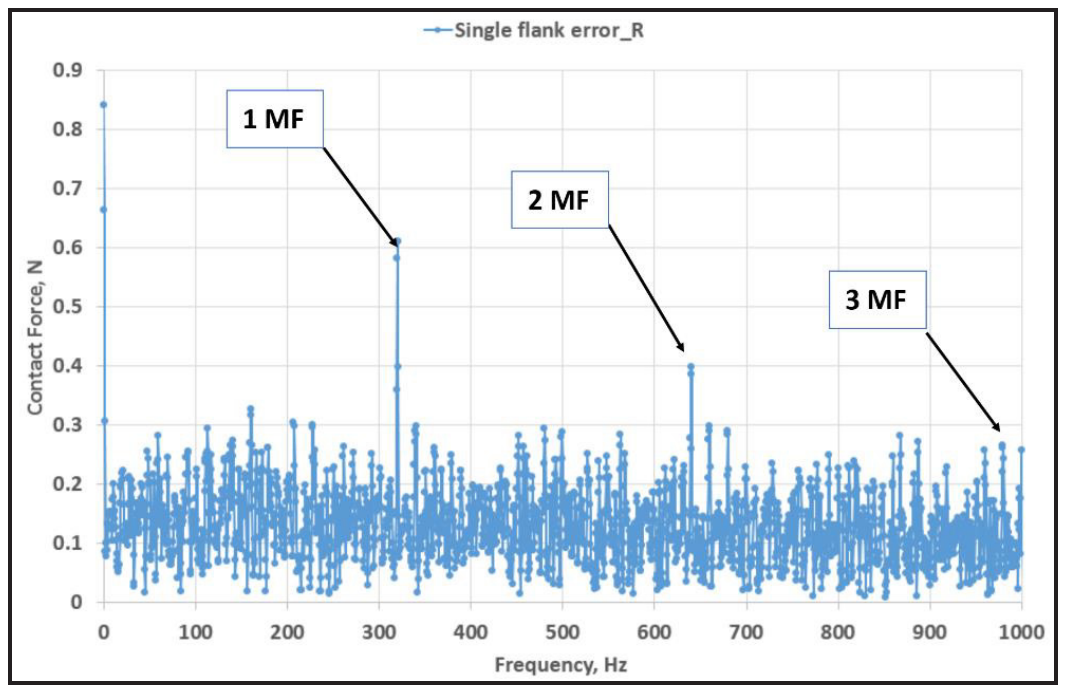

Fig. 5: Frequency domain signal of single flank error induced planetary gearbox

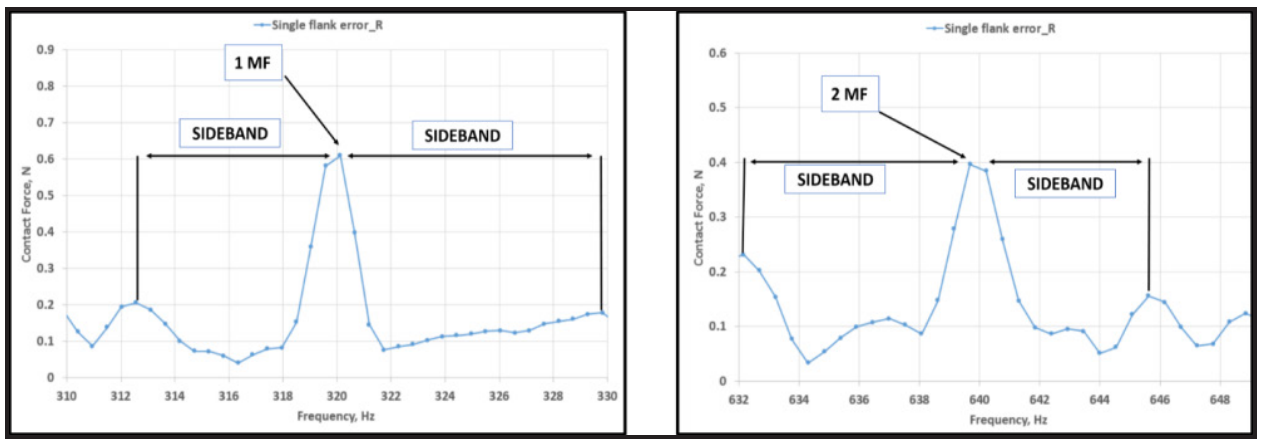

Fig. 6. Sidebands in the vicinity of mesh frequencies

These simulation result addresses the presence of single flank error and its effect on dynamic condition. Analysis of these results are discussed in section 7.

\section{Error modelling and rigid body simulation for transmission error}

When the planetary gearbox transfers motion from input to output shaft, it should be of uniform motion without any losses. This is possible only in an ideal condition which is unrealistic in nature. Various type of errors related to gear manufacturing have significant influence on these losses. These losses are generally known as transmission error of a 
gearbox. From literature, it is inferred that there are challenges in addressing, how these errors affect the transmission error of a planetary gearbox.

Transmission error is the difference between theoretical and measured angular velocity of any gear mesh. The term transmission error is represented in form of a mathematical expression as shown in eqn. 6 ,

$$
\text { Transmission Error }=\theta_{\mathrm{s}}-(\mathrm{i}) * \theta_{\mathrm{c}}
$$

Where:

$\theta_{\mathrm{s}}$ is angular rotation of input gear (sun gear), rad

$\theta_{\mathrm{c}}$ is angular rotation of output gear (Carrier gear), rad

$\mathrm{i}$ is the velocity ration of the planetary gear

In this section, transmission error is studied without considering the effect of tooth defection. To simulate the transmission error, the sun gear (input angle) is rotated by 3.14 $\mathrm{rad} / \mathrm{s}$ and simulated for 1 second with 5000 steps. It infers that sun gear rotates by $3.14 \mathrm{rad}$ in a second with 5000 equal interval steps. According to gear ratio, angle rotated by carrier should be $1 / 5$ th of the input angle. The difference between the input and output angle gives the transmission error. The complete simulation is carried out under no-load condition. The variation of transmission error in planetary gearbox due to single flank error is shown in Fig. 7.

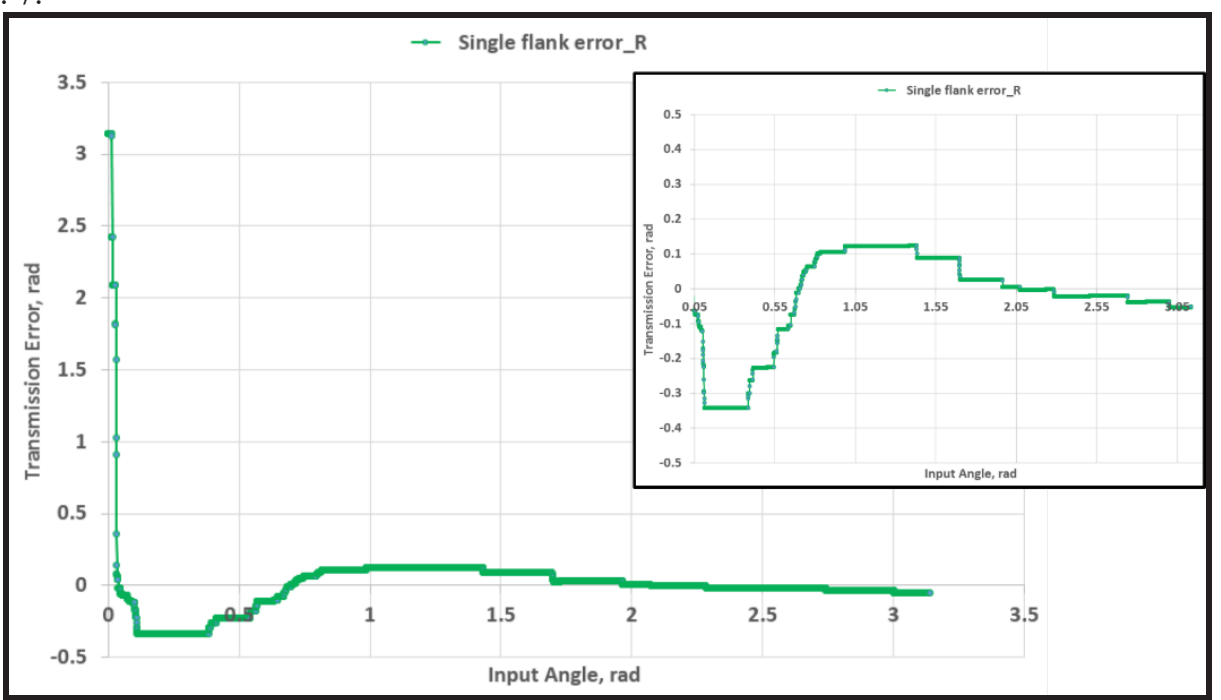

Fig. 7. Transmission error variation

\section{Flexible body simulation of single flank error}

In previous section, the effect of single flank error on transmission error is studies through rigid body analysis, i.e, without considering any tooth deflection. In this section, the designed planetary gearbox is analysed for transmission error by considering gear tooth deformation. The planetary gearbox is modelled as plane stress condition as there are no stresses developed in out plane section as shown in Fig. 8. In addition to this, shafts are assumed to be rigid.

The element size arrived from the convergence study is $0.4 \mathrm{~mm}$. CPS4R [10] element is selected, which has 4-node bilinear, reduced integration with hourglass control. This 
element is a 3 DOF with two translational and one rotational degree of freedom. The material property assigned is as provided in table 2 .

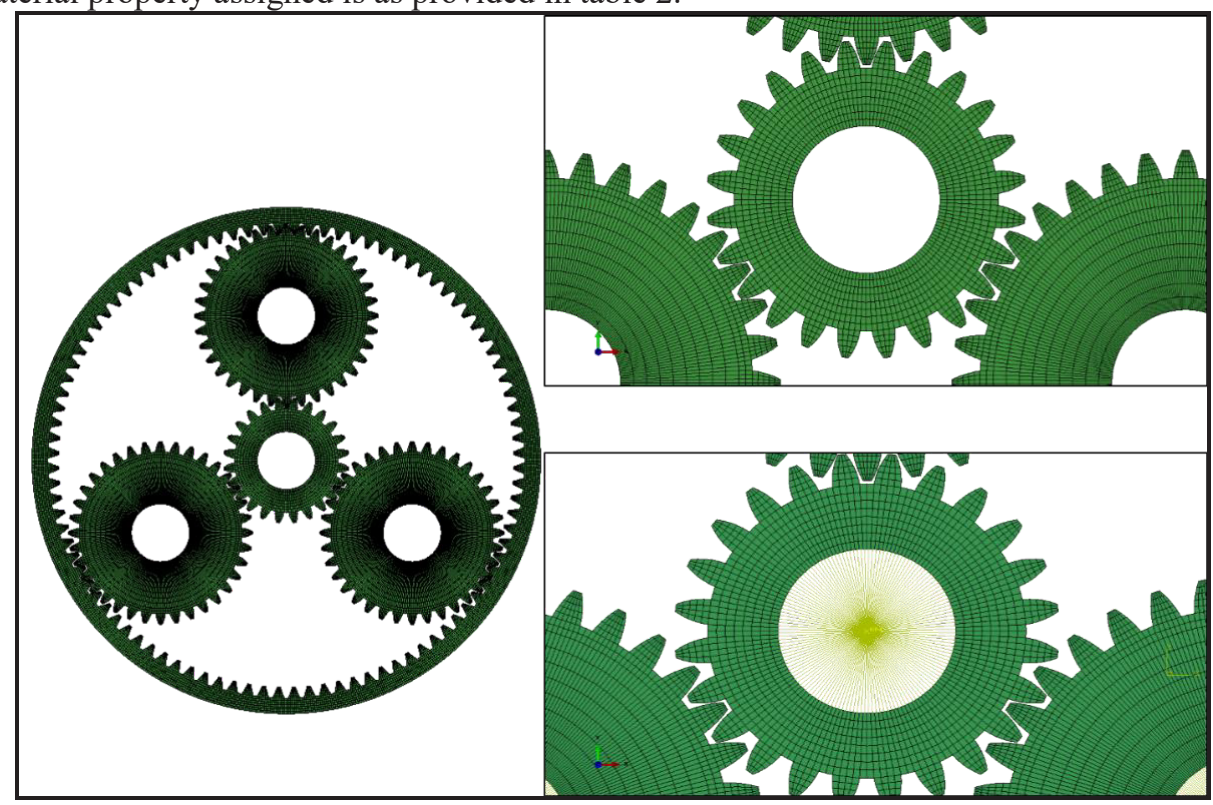

Fig. 8. Finite element model of planetary gear

Table 2. Steel material properties

\begin{tabular}{|c|c|}
\hline Material Properties & Values \\
\hline Young`s Modulus & $210000 \mathrm{~N} / \mathrm{mm}^{2}$ \\
\hline Density & $7.58 \mathrm{e}-09$ tonne $/ \mathrm{mm}^{3}$ \\
\hline Poisson`s ratio & 0.3 \\
\hline
\end{tabular}

Two types of forces arise during gear teeth contact: normal and tangential. For tangential component, a co-efficient of friction 0.03 is applied through penalty method. In the case of normal contact, a hard contact is applied with no separation of mating surface.

As multiple tooth contact are involved, a non-linear quasi-static analysis is carried out. Reference points representing the rigid shafts are used for applying loads and fixing boundary condition in simulation. The ring gear is fixed, input torque and resisting torque are applied on input and carrier arm. Planet gears are allowed to rotate about the axis of sun gear. One pitch rotation angle is provided as input for the sun gear. The complete analysis is divided into two step process. Each step is further divided into 1000 sub-step with 1e-5 increment value.

Input and output angles are measured during post processing of results. Difference between them gives the transmission error due to fault induced in planetary gearbox and is shown in Fig. 9. 


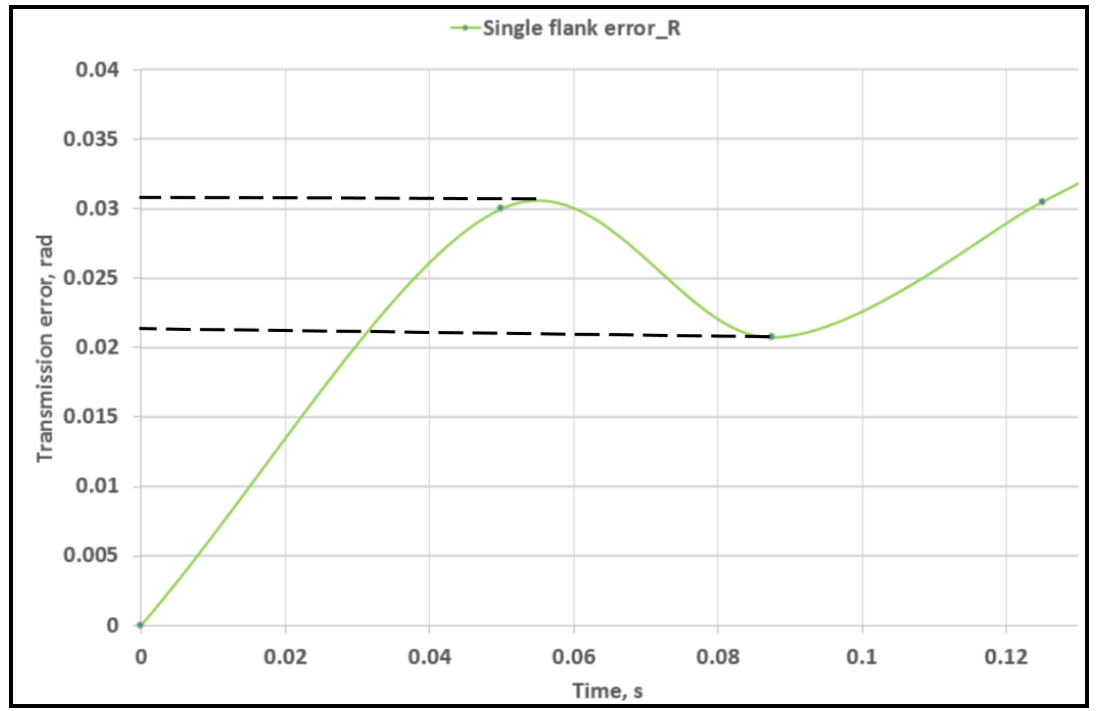

Fig. 9. Transmission error plot of error induced planetary gearbox

\section{Results and discussion}

In this section, the effect of single flank error on dynamic response of planetary gearbox is analyzed using time and frequency domain signal. The variation in tooth thickness due to single flank error is shown in Fig. 2. This variation is due to the single flank error of $43 \mu \mathrm{m}$ modelled on single gear tooth. The single flank error causes the variation of contact forces which is presented in Fig. 4. This is due to the fact that there is a delay in modelled gear tooth to come in contact with other meshing tooth.

When a time domain signal is converted to a frequency domain signal, the single flank error does not cause any change in mesh frequency and its harmonics. But when observed closely around the mesh frequencies, small peaks are present and these peaks are known as sidebands. These sidebands are shown in Fig. 6 and frequencies are known as pass frequencies. These pass frequencies are exactly $7 \mathrm{~Hz}$ on either side of mesh frequencies. These frequencies are calculated based on a literature [11].

A close observation of Fig. 2 shows the clearance between the gears are not uniform, this causes non-uniformity in motion. This non-uniformity in motion is shown in Fig. 7. In an ideal condition, the transmission error should be zero. On the other hand, the presence of single flank error varies the transmission error from -0.35 to 0.12 radians for an input of 3.1415 radians without considering any tooth deflection. This shift is due to the variation between the teeth as shown in Fig. 2 .

The transmission error in a planetary gearbox is analysed using flexible body analysis with considering tooth deflection. In this case, the transmission error varies from -0.021 radian to +0.031 radians for an input of one pitch rotation.

\section{Conclusion}

The effect of single flank error on uniform transfer of motion is analyzed through rigid and flexible body analysis. The single flank error of DIN class 8 is modelled and simulated for frequency domain signal through rigid body analysis. In the presence of $43 \mu \mathrm{m}$ single flank error is indicated by equally spaced sidebands around the mesh frequencies and its 
harmonics. Amplitude of the sideband depends on intensity of error. Simulation results of mesh frequencies and sidebands are in good agreement with analytical calculation of existing literature [10]. And the transmission error value varies from -0.3 to 0.12 radians. Similarly, in flexible body analysis the transmission error value varies from 0.021 to 0.031 radians. Finally, it is concluded that the presence of time invariant single flank error has significant influence on planetary gearbox dynamics and transmission error. And transmission error in a planetary gearbox is significantly increases with tooth deflection and manufacturing error.

\section{References}

1. J. Lin, R. G. Parker., J. Vib. and Acoust., 3, 121 (1999)

2. R. G. Parker, S. M. Vijayakar, \& T. Imajo., J. Sound Vib., 3, 237 (2000)

3. W. Bartelmus, J. Mech. Sys. and Sig. Proc., 5, 5 (2001)

4. W. Bartelmus, F. Chaari, R. Zimroz \& M. Haddar., E. J. Mech. A Solids., 4, 29 (2010)

5. C. Zhu., X. Y. Xu., T. C. Lim., X. S. Du \& M. Y. Liu, J. Mech. Engg. Sci., 1, 227 (2012)

6. F. Charri, T. Fakhfakh \& R. Hbaieb. J. Adv. Manu. Tech., 27, (2006)

7. A. Bodas, A. Kahraman, JSME Series C, 47(3), (2004)

8. DIN 3961, Tolerance for cylindrical teeth, Germany (2002)

9. D. D. A. Browski, J. Adamczyk, \& H. P. Mora, Diagnostyka- Applied Structural Health, Usuage and Condition Monitoring. 2, 62 (2012)

10. ABAQUS/CAE 6.11 User's Manual

11. Y. Lei, J. Lin, M.J. Zuo \& Z. He., J. Int. Mea. Confed., 1, 48 (2014) 